\title{
Fermented Vegetables, a Rich Repository of Beneficial Probiotics-A Review
}

\author{
Sajad Ahmad Mir ${ }^{*}$, Jeelani Raja and Masoodi FA
}

Department of Food Science \& Technology, University of Kashmir, Srinagar, India

*Corresponding author: Sajad Ahmad Mir, Department of Food Science \& Technology, University of Kashmir, Srinagar, India, Tel:+ 97-970-50619; E-mail: mirsajad004@gmail.com

Received date: January 04, 2018, Accepted date: January 25, 2018, Published date: February 03, 2018

Copyright: ( 2018 Mir SA, et al. This is an open-access article distributed under the terms of the Creative Commons Attribution License, which permits unrestricted use, distribution, and reproduction in any medium, provided the original author and source are credited.

\begin{abstract}
Fermentation is an old age biotechnological technique for preservation of vegetables, which has paved pathway towards nutritional and functional value of the foods. It not only preserves food for long period of time but also increases functional, nutritional and sensory features of food commodities. Fermentation is the outcome of the the microbes which grow in the food commodity with the passage of time. Leuconostoc mesenteroides and related LAB, including Weissella and other Leuconostoc spp. are important in the initiation of the fermentation of many vegetables. Fermented foods are supposed to be rich in the health beneficial probiotics. Fermented vegetables are low-calories foods as they contain considerably lower quantities of sugars compared to their raw counter parts. They are source of dietary fibre, which impedes the assimilation of fats and regulates peristalsis in the intestines and valuable source of vitamin C, B group vitamins, phenolics and many other nutrients.
\end{abstract}

Keywords: Fermentation; Vegetables; Microbes; Probiotics; Health beneficial

\section{Introduction}

The term probiotics was first coined by Kollath and Vergio [1]. Probiotics were originally defined as microorganisms who promote the growth of other microbes [2,3]. Probiotics have been re-defined a number of times. Probiotics as a live microbial, feed supplement which beneficially affects the host animal by improving its intestinal microbial balance [4]. Probiotics are mono or mixed cultures of live microorganisms which when applied to animal/man, beneficially affect the host by improving the property of the indigenous flora [5]. Probiotics have been defined by the German Federal Institute for health and consumer protection and veterinary medicine as specific live microorganisms which reach the intestinal tract in active form and in sufficient number to positively affect the health of the host. The most recent definition of the probiotics by [6] states that probiotics are live microorganisms which when administered in adequate amounts $\left(10^{6}-10^{7} \mathrm{CFUg}^{-1}\right.$ of food) confer health benefits on the host. Any bacterial strain, before being used as probiotics, should be screened for various imperative characteristics in order to satisfy the regulatory bodies as well as ensure consumer acceptance. The ideal probiotic bacteria possess all such characteristics like ability to colonize human intestine, resistance to bile and low $\mathrm{pH}$, antibiotic susceptibility and antimicrobial activity $[7,8]$.

Two classes of lactic acid producing microorganisms have been recommended for human application. These include the bifidobacteria and lactic acid bacteria including species of Enterococcus, Lactobacillus, Lactococcus, Leuconostoc, Pediococcus and Streptococcus [9]. Some yeast strains such as Saccharomyces cerevisiae and Saccharomyces boulardii also have shown a probiotic influence on human intestinal flora [10].

\section{An Overview of Indian Fermented Vegetable Products}

\section{Gundruk}

Gundruk is a pickled product of Nepal, which is obtained from fermentation of leafy vegetables. The production process is similar to that of sauerkraut, however no salt is added to the shredded leaves prior to fermentation. Gundruk fermentation is usually dominated by Pediococcus and Lactobacillus spp., L. cellobiosus and L. plantarum being the initiators of fermentation. During the course of fermentation the $\mathrm{pH}$ drops slowly to a final value of 4 and total acidity as lactic acid increaes to about $1 \%$ [11]. Gundruk may be served as a side dish (soup/pickle) and also as an appetizer.

\section{Sinki}

It is a sour pickled radish taproot that is traditionaly consumed in India, Nepal and Bhutan either as a pickle or as a base for soup [12]. Sinki has been shown to act as an effective appetizer, helps cure diarrhea and relieves stomach pain. Sinki fermentation is carried out by $L$. fermentum, L. brevis and $L$. plantarum decreasing the $\mathrm{pH}$ from 6.7 to 3.3.

\section{Khalpi}

Khalpi is a fermented cucumber product of Nepal which is usually eaten as pickle after mixing with mustard oil, salt and powderd chillies. The bacteteria like L. plantarum, L. brevis and Leuconostoc fallax are usually involved in khalpi fermentation.

\section{Inziangsang}

It is a traditional fermented leafy vegetable product which is consumed in Northeast India, especially in Nagaland and Manipur. It is made from mustard leaves and the production process is similar to that of gundruk [13]. Inziangsang can be stored for a year or more at 
room temperature. It is usually consumed as a soup. Fermentation usually involves L. plantarum, L. brevis and Pediococcus [12].

\section{Soidon}

Soidon is a fermented product of bamboo shoots and is popular in Manipur. Only the tips are used for fermentation while the rest portions are removed. For the enhancement of flavor, leaves of Roxb (Garcinia pedunculata) may be added during fermentation. Lactobacillus brevis, Leuconostoc fallax and Lactococcus lactis are the predominant organisms involved in its fermentation [12].

\section{Goyang}

Goyang is a fermented vegetable product of Sikkim and Nepal which is prepared from the leaves of magane-saag (Brassicaceae). Lactobacillus plantarum, Lactobacillus brevis, Lactobacillus lactis, Enterococcus faecium, Pediococcus pentosus and yeast like candida spp. are the main strains that carry out fermentation.

\section{Mesu}

Mesu is a traditional fermented bamboo shoot product of Darjeeling hills and Sikkim. It is made from the defoliated and finely chopped young edible shoots of choya bans (Dendrocalamus hamiltonii), Karati bans (Bambusa tulda) and Bhalu bans (Dendrocalamus sikkimensis). The typical taste and mesu flavor indicate the completion of fermentation. Mesu is usually consumed as a pickle. Mesu pickle, after mixing with edible oil chillies and salt can be stored for several months without refrigeration. Lactobacillus plantarum, Lactobacillus brevis, Lactobacillus curvatus, Leuconostoc citreum, Pediococcus pentosaceus are the dominant origanisms involved in fermentation [14].

\section{Soibum}

Soibum is a fermented bamboo shoot product of Manipur with whitish colour, faint aroma and sour taste. It is made from succulent bamboo shoots of Dendrocalamus hamiltonii, D. sikkimensis and $D$. giganteus, Bambusa tulda and B. balcona. Soibum is usually consumed as a side dish. The bacterial flora like $L$. plantarum, $L$. brevis, $L$. coryniformis, L. delburkii, Leuconostoc fallax, Lactococccus lactis, $L$. mesenteroides, Entreococcus durans, Streotococcus lactis, Bacillus subtillis, B. licheniformis, B. coagulans and yeast like Candida, Saccharomyces and Torulopsis are mostly involved in its fermentation $[15,16]$.

\section{Ekung}

Ekung is a fermented bamboo tender shoot product of Arunachal pradesh. It is made from young bamboo tender shoots of Dendrocalamus hamiltonii, D. giganteus, Bambusa balcooa, Phyllostachys assamica, B. tulda.

\section{Anishi}

Anishi is a fermented vegetable product of Nagaland. It is made from Yam leaves and is usually used as a condiment [17].

\section{Lung-Siej}

Lung-Siej is another fermented bamboo shoot product of Meghalaya. It is made from the bamboo species Dendrocalamus hamiltonii. The bamboo shoots fermented in bottles have a better shelf life compared to that of bamboo cylinders. Lung-siej is usually consumed as curry mixed with meat and fish [18].

\section{Fermented garlic}

A number of garlic based products are manufactured in the world which includes garlic oil, garlic flakes, fried garlic slices, baked garlic slices, garlic salt and garlic paste. Blanching and fermentation of garlic has also been studied for their beneficial effects [19]. Due to its beneficial health effects, which include anti-microbial activity, antioxidant activity, garlic has attracted much more research interest. It is used as condiment in the preparation of dressed olive oils in Spain. It is used an agent for prevention and treatment of cardiovascular, atherosclerosis, hyperlipidemia, thrombosis, and diabetes. Both raw and fermented garlic increases the production of Nitric Oxide Synthase (NOS), a key in the lowering of blood pressure. Garlic enhances the immune system of some people living with HIV by increasing the number of natural killer cells which not only destroy white cells that are infected by viruses but also those which are cancerous. Fermented garlic not only lowers down the cholesterol level but triglycerides as well.

\section{Fermented Vegetables as a Source of Probiotics}

Fermentation is the oldest biotechnological method to preserve vegetables. The preservation of vegetables by fermentation was recognized before recorded history [20]. Vegetables belong to the category of perishable foods. Consequently different preservation techniques like refrigeration, freezing and canning have been developed to prolong their shelf life. However, in underdeveloped and developing countries such preservation methods are inaccessible making them to rely on the well adapted and proven natural preservation methods. Fermentation remains the most-oldest biotechnological and practiced method to preserve vegetables, which is accompanied by changes in organoleptic properties and enhancement of nutritional quality [21]. Lactic acid bacteria (LAB) are naturally present on the various parts of the vegetables, which cause spontaneous lactic acid fermentation once the favourable conditions of water activity, salt concentration, anaerobiosis and temperature are provided. Leuconostoc, lactobacillus and pediococcus are the most common genera involved in lactic acid fermentation of vegetables. The presence of the species of genus Lactococcus next to these three genera has been confirmed by [22]. Several species of Weisella like W. cibaria and $W$. paramesenteroides have also been reported in some fermented vegetables such as cabbage and mustard [23,24]. Researches have concluded that the LAB derived from plants are similarly resistant to artificial gastric juices and bile as are the animal derived LAB and are therefore considered as promising probiotics for human consumption. Thus, it is supposed that fermented vegetables as such can serve as an alternative source of probiotic.

Increasing demand for vegetarian foods has been challenging the food industry to manufacture high quality functional products [25]. In addition to this lactose intolerance of a larger segment of world population together with the undesirable cholesterol content of fermented dairy products has opened a window of opportunities for developing non-dairy probiotic products [26]. This calls for alternative sources of LAB such as fruits, vegetables, legumes and cereals [27] which by appropriate technological handling may serve as probiotic vectors. There is unfortunately scare information available about the vegetables in terms of delivery of probiotic strains. 
Various efforts have been made by the researchers on the isolation of LAB with probiotic properties from the fermented vegetables. Pediococcus pentosaues strain MP12 and Lactobacillus plantarum strain LAP6, isolated from pickled cabbage, have been found to show antagonist activity against Salmonella spp. in mice where both the strains were able to adhere to the mouse intestinal epithelium [28]. Similarly Lactobacillus plantarum CO6 and Lactobacillus acidolphillus C11, that were isolated from pickled cabbage were found to satisfactorily adhere to duodenum, tolerate the gut biotic stress-gastric juices and bile salts, exhibit antimicrobial activity against grampositive and negative pathogens and $\beta$-galactosidase activity [29]. Lactococcus lactis has been isolated from sauerkraut that produces bacteriocin-nisin. LAB from fermented cucumber produce bacteriocin-plantaricin C19 against Listeria grayi, while as LAB from fermented olives are the producers of bacteriocins against Weissela mesenteroides [30]. Various in vitro studies have reported that LAB isolated from fermented olives are capable of adhering to epithelial cells IPEC-J2 of porcine jejune and produce bacteriocins against Helicobacter pylori, Propionibacterium spp. and Clostridium perfringens [31]. Lactobacillus starins isolated from fermented carrot produce bacteriocins against $E$. coli, $S$. aureus and B. cereus [32]. L. sakei isolated from fermented chinese cabbage produces bacteriocin Sakacin C2 which strongly inhibits E. coli. And S. aureus ATCC 63589. Also $L$. plantarum IB2, isolated from inziangsang is the producer of a bacteriocin against Staphylococcus aureus S1. LAB strains isolated from fermented vegetable pickles have been found to be effective against type-I allergies, by increasing the production of Th1/Th2 balance and reducing the production of IgE [33]. These studies suggest that LAB strains isolated from various fermented vegetable products can serve as a good alternative for the supply of health-benefiting probiotics.

\section{Health benefits of fermented vegetables due to probiotics}

Consumption of fermented vegetables has been associated with a range of health benefits arising from the growth and activity of probiotic lactic acid bacteria during fermentation. The exact mechanisms behind the health promoting properties of probiotics are not still completely understood. However the most beneficial effects of the probiotic are related to pathogen interference, exclusion or antagonism, immune modulation, anticarcinogenic and antimutagnic activities, alleviation of lactose intolerance symptoms, reduction in serum cholesterol levels, reduction in blood pressure, prevention of bacterial vaginosis and urinary tract infection, maintenance of mucosal integrity and improved periodontal health. The various health benefits from fermented vegetables are discussed as follows.

Anticancerous properties: LAB from various fermented vegetables have been shown to posses anticancerous and antimutagenic properties. Cancer preventative activity of kimchi and inhibition of the proliferation of human cancer cell lines for 3 weeks by the extract of fermented kimchi has been reported earlier [34]. It was seen that the leukemia cells treated with kimchi extract showed increased apoptosis and decreased mitochondrial transmembrane potential [35], however, the normal cells remained unaffected. The anticarcinogenic effect of kimchi LAB has been shown to be due to the suppression of the activity of carcinogen activating enzymes such as azoreductase, nitroreductase, 7 - $\alpha$-dehydrogenase, $\beta$-glucosidase and $\beta$-glucuronidase and neutralization of cancer causing agents. The anticancer property of probiotic like $L$. reuteri and down regulation of nuclear-factor-kappa B dependent gene products which inturn regulate cell proliferation and survival has been investigated earlier [36]. Besides it, L. reuteri was found to suppress TNF induced NF- $\mathrm{B}$ activation and slowed down the growth of cancer cells. Antitumourigenic activity of the exopolysaccharide producing $L$. acidophillus and L. rhamnosus against colon cancer cells by the activation of autophagic cell death by inducing Beclin-1 and GRP78 and Bcl-2 and Bak has been investigated [37], also $L$. acidophillus and $L$. casei can be used as adjuvants in anticancer chemotherapy due to their enhanced apoptosis induction capacity of fluorouracil in colorectal carcinoma cell line LS513 [38]. The antitumour properties of LAB are mainly by the activation of immune cells to fight against tumor cells. Earlier Studies have shown an increase in TNF- $\alpha$ interferon- $\gamma$ and regulatory cytokine IL-10 due to the probiotic LAB [39].

The probiotic bacteria were reported to show high degree of survival after genotoxin exposure [40]. 8 out of 11 probiotic bacteria showed a strong genotoxicity inhibition and the strain Enterococcus faecium AdF2 and Lactobacillus plantarum AdF10 were reported to have maximum antigenotoxicity, comparable to that of the refrence strain Lactobacillus rhamnosus G G (ATCC 53103). Inhibition of genotoxicity has been suggested to be due to the binding of genotoxins to the probiotics.

Anticholesterolemic effects: It is well established fact that high-total cholesterol and LDL-cholesterol levels are the major risk factors for coronary heart diseases and it is the leading cause of death in West [41]. Consequently it becomes important to devise new methods to reduce the serum cholesterol levels. Recently LAB from fermented foods have been reported to show various anticholesterolemic effects.

It was reported that after 7 days of kimchi intake, the concentrations of fasting blood glucose (FBG), total glucose, total cholesterol and LDL was significantly dropped. Various studies have suggested mechanisms on the lipid lowering effect of kimchi. LAB involved in the fermentation of kimchi have been reported to posses cholesterol lowering activity either by binding the cholesterol in their cell wall, decomposing the cholesterol for assimilation or deconjugation of bile acids.

Bioactive compound of kimchi, when administered into hypercholesterolemic rabbits showed a decrease in plasma cholesterol and LDL-cholesterol within 4 days after treatment [42]. Additionally other ingredients of kimchi such as Chinese cabbage, hot red peppers, garlic, ginger etc. have been shown to contribute to cholesterol lowering activity. These ingredients contain certain bioactive compounds like $\beta$-sitosterol (Chinese cabbage), S-methylcysteinsulfoxide and S-allylcysteinsulfoxide (garlic) and capsaicin (red peppers) that are believed to contribute to anticholesterolemic effect. $\beta$-sitosterol being a phytosterol competes with the dietary cholesterol for intestinal absorption [43]. Sulphur compounds in garlic promote the secreation of hormones like adrenalin and glucagon and hence stimulate lipolysis or suppress enzyme activities that are responsible for cholesterol synthesis. Allicine has been reported to inhibits the activity of Acetyl-CoA synthetase and/or 3-hydroxy-3-methyl-glutaryl CoA reductase [44]. Capsaicin elevates the activity of $7 \alpha$-hydroxylase and thereby stimulating the secreation of cholesterol to extra-circulation as bile [45].

Lactobacillus plantarum is one of the most frequent LAB strain to occur in fermented vegetables and has been isolated from fermented cucumbers, cabbage, eggplant, tomato, red-beet etc. Also L. plantarum has been evalvated as a potential probiotic with cholesterol lowering effect. It was reported that feeding of L. plantarum $\mathrm{PH} 04$ to hypercholestrolemic mice at doses of $10^{7} \mathrm{CFU} /$ mouse/day for 14 days 
resulted in $7 \%$ lower total cholesterol and $10 \%$ lower serum triglycerides compared to the control mice. The mechanism behind the cholesterol lowering effect has been suggested to be the deconjugation of bile salts by the enzyme bile salt hydrolase [46]. Which in turn promotes the excreation of bile salts and leads to an increased synthesis of bile salts from serum cholesterol or by decreasing the solubility of cholesterol and consequently reducing the uptake from gut.

Immunomodulatory effect: Clinical and animal studies have provided evidences that $\mathrm{LAB}$ can modify the immune response of the host [47]. Earlier 159 strains of lactic acid bacteria from traditional Taiwan fermented mustard for evalvation of their immunopotentiating activity on a murine macrophage cell line RAW 2647 has been studied [48]. Of all, the strain L. plantarum B0040, Weissella cibaria B0145 and B0110 were able to activate the macrophage and showed a pronounced increase in the level of nitric oxide, tumor necrosis factor- $\alpha$ and interleukin-6. Earlier studies has reported the modulation of immune response by $P$. pentosaceus NB-17 by inducing the production of cyotokines in the mouse spleen cells and was also able to survive the gastric conditions of rats [49].

The probiotic potential of fermented vegetable derived LAB, $L$. plantarum 10HK2 was investigated and was reported to show antibacterial activity against pathogens and immune modulating effects on murine macrophage cell lines. A reduced number of enteric Salmonella and Shigella species was reported in the mice fed with $L$. plantarum as compared to the controls for upto 4 weeks. The effect on immunoglobulin (Ig) and cytokine production was investigated by oral administration of the live and heat killed A17 to OVA sensitized mice. The immune responses of $\mathrm{A} 17$ by IFN- $\gamma$ production,were verified using human peripheral blood mononuclear cells. It was reported that A17 modulated the OVA-induced allergic effects by modulating B-cell and T-cell responses. The former through increased IgE production and elevated OVA specific IgG2a production while as the later through increased IFN- $\gamma$ production and decreased IL- 4 production. A17 also down regulated the mRNA expression of nucleotide-binding oligomerization domain protein, NOD-1, NOD-2 and Toll like receptor (TLR)- 4 .

Lactobacillus strains like L. plantarum CJLP133, L. plantarum CJLP243, L. plantarum CJNR26 and L. gasseri CJMF3 has been isolated from Korean fermented vegetables and investigated for their capacity to modulate cellular and humoral immune responses [50]. It was found that 8 weeks feeding of the tested lactobacilli did not affect the weight of and cell numbers in the mice spleen. However the strains CJLP133 and CJLP243 were found to increase the T lymphocyte population while as the strains CJNR26 and CJMF3 increased the B lymphocyte population in the spleenocytes treated with concanavalin A. These results indicate that CJLP133 and CJLP243 have immune stimulating activity via increased T-cell activation. While as CJNR26 and CJMF3 exhibit immunopotentiation through enhancement of Bcell activation. The probiotic potential of Lactobacillus sakei probio 65, isolated from kimchi, for treating allergic dermatitis has been reported [51]. The strain was administered to allergen-induced mice, trigerred with 1-chloro-2,4-dinitrobenzene. It was reported that the mice receiving $L$. sakei probio 65 treatment recovered rapidly compared to control mice through the regulation of Ig and IL-4 levels in the sensitized mice.

The results showed that the strain offered $100 \%$ protection against infection with influenza A viruses, prevented significant weight loss and lung viral loads were lowered in the mouse. High levels of cytokine-IL-12 and IFN- $\gamma$ were found in the mice treated with DK119.
The results showed that DK119 exhibited antiviral effects on influenza virus infection through the modulation of host innate immunity of dendritic and macrophage cells and cytokine production.

\section{Shelf life of probiotics in fermented vegetables}

Plant tissues consist of intricate internal cells, intercellular spaces, pores and capillaries. Although the intact cell wall acts as an effective barrier against the microbial action. However the microorganisms which tend to enter the plant tissue via lenticels and tissue lesions are entrapped. Once the microbes cross all the barriers, there growth is facilitated by the release of nutrients through the holes formed in the plant tissues. The release of nutrients is further increased by steps like cutting and peeling, thereby allowing the vegetables to be used as a carrier of probiotics. It is further suggested that like in fermented dairy products certain LAB strains show high survival rates in the fermented plant materials (table olives and artichokes) or even higher than the dairy probiotics. This has been attributed to the microstructure of plants that provides favourable and protective conditions for the bacterial survival. Besides the cell wall is solid and resistant enough to allow the bacterial adaptation to the harsh environmental conditions of the plant matrices such as high osmotic pressure, poor nutrient profile, presence of antimicrobial compounds, etc. [33].

\section{Conclusion}

Fermentation is a cost effective technique utilized for the preservation of the perishable vegetables. It not only presreves the food commodity for off season consumption, but also increases its nutritional and functional value. Fermented foods are cherished for their specific taste and flavor. Fermented foods are laid with large number of probiotic microorganisms, which have proven health benefical effect. However further scientific approaches are required in order to fully explore the vast benefits of the fermented foods.

\section{Acknowledgment}

The authors are thankful to the Department of Biotechnology, Government of India, for its financial support.

\section{Conflict of Interest}

There is no financial interest or any conflict of interest exists.

\section{References}

1. Holzapfel WH, Schillinger U (2002) Introduction to pre- and probiotics. Food Res Int 35: 109-116.

2. Vergin F (1954) [Antibiotics and probiotics]. Hippokrates 25: 116-119.

3. Lilly DM, Stillwell RH (1965) Probiotics: growth promoting factors produced by microorganisms. Science 147: 747-748.

4. Fuller R (1989) Probiotics in man and animals. J Appl Bacteriol 66: 365-378.

5. Havenaar R, Ten Brink B, Huis in't Veld JHJ, (1992) Selection of strains for probiotic use. In: Fuller, R. (Ed.), Probiotics, the scientific Basis. Chapmann \& Hall, London, pp. 209-224.

6. FAO/WHO (2002) Guidelines for the evaluation of probiotics in food. Joint FAO/Whop working Group.

7. Lee YK, Salminen S (2009) Characterization of probiotic properties in Bifidobacterium and Lactobacillusstrains. Probiotic microorganisms. Handbook of probiotics and prebiotics (2nd ed.). 19-24. 
8. Kruszewska D, Lan J, Lorca G, Yanagisawa N, Marklinder I, et al. (2002) Selection of lactic acid bacteria as probiotic strains by in vitro tests. Microb Ecol Health Disease 29: 37-49.

9. Martins EMF, Ramos AM, Vanzela ESL, Stringheta PC, de Oliveira Pinto CL, et al. (2013) Products of vegetable origin: A new alternative for the consumption of probiotic bacteria. Food Res Inter 51: 764-770.

10. Sourabh A, Kanwar SS, Sharma OP (2011) Screening of indigenous yeast isolates obtained from traditional fermented foods of Western Himalayas for probiotic attributes. J Yeast Fungal Res 2: 117-126.

11. Karki T (1986) Some Napalese Fermented foods and beverages. In Traditional foods: Some products and technologies. Mysore: central Food Technological Research Institute.

12. Tamang JP (2009) Himalayan Fermented Foods: Microbiology, Nutrition and Ethnic Values, CRC Press, New Delhi, India.

13. Yan PM, Xue WT, Tan SS, Zhang H, Chang XH (2008) Effect of inoculating lactic acid bacteria starter cultures on the nitrite concentration of fermenting Chinese paocai. Food Control 19: 50-55.

14. Tamang JP, Sarkar PK (1996) Microbiology of mesu, a traditional fermented bamboo shoot product. Int J Food Microbiol 29: 49-58.

15. Giri SS, Janmejay LS (1987) Microbial and chemical contents of the fermented bamboo shoot soibum. Frontier Bot 1: 89-100.

16. Sarrangthem K, Singh TN (2003) Microbial bioconversion of metabolites from fermented succulent bamboo shoots into phtosterols. curr sci 84 : 1544-1547.

17. Agrahar-Murungkar D, Subbulakshmi G (2006) Preparation techniques and nutritive value of fermented foods from the khasi tribes of Meghalaya. Ecol Food Nutr 45: 27-38.

18. Mao AA, Odyuo N (2007) Traditional fermented foods of the Naga tribes of Northeastern India. Ind J Trad knowled 6: 37-41.

19. Raja J, Mir SA, Masoodi FA (2016) Effect of dry salt and brine on the fermentation and colour of blanched garlic. J Nutr Food Sci 6: 484.

20. Fleming HP, Kyung KH, Breidt F (1995) Vegetable fermentations. In: Rehm HJ, Reed G. Biotechnology, volume.9. Enzymes, biomass, food and feed, 2nd edition. New York: VCH.

21. Tamang JP (2010) Diversity of fermented foods. In: Tamang JP, Kailasapathy K (Eds.), Fermented Foods and Beverages of the World. CRC Press, New York, pp. 41-72.

22. Yasuhiro I, Latiful BM, Susumu K, Shinichi K (2005) Bacteria in traditional fermented vegetables produced in northern part of Vietnam. Jap J Food Microbiol 22: 103-111.

23. Chao SH, Wu RJ, Watanabe K, Tsai YC (2009) Diversity of lactic acid bacteria in suan-tsai and fu-tsai, traditional fermented mustard products of Taiwan. Inter J Food Microbiol 135: 203-210.

24. Jung JY, Lee SH, Lee HJ, Seo HY, Park WS, et al. (2012) Effects of Leuconostoc mesenteroides starter cultures on microbial communities and metabolites during kimchi fermentation. Inter J Food Microbiol 153: 378-387.

25. Heenan CN, Adams MC, Hosken RW, Fleet GH (2004) Survival and sensory acceptability of probiotic microorganisms in a non-fermented frozen vegetarian dessert. LWT Food Sci Technol 37: 461-466.

26. Granato D, Branco GF, Nazzaro F, Cruz AG, Faria JAF (2010) Functional foods and non-dairy probiotic food development: Trends, concepts and products. Compreh Rev Food Sci Food Safety 9: 292-302.

27. Luckow T, Delahunty C (2004) Which juice is healthier? A consumer study of probiotic non-dairy juice drinks. Food Qual Pref 15: 751-759.

28. Chiu H, Tsai C, Hsih HY, Tsen HY (2008) Screening from pickled vegetables the potential probiotic strains of lactic acid bacteria able to inhibit the Salmonella invasion in mice. J Appl Microbiol 104: 605-612.

29. Wang CY, Lin PR, Ng CC, Shyu YT (2010) Probiotic properties of Lactobacillus strains isolated from the faeces of breast-fed infants and Taiwanese pickled cabbage. Anaerobe 16: 578-585.

30. Delgrado A, Brito D, Peres C, Noe-Arroyo F, Garrido-Fernandez A (2005) Bacteriocin production by Lactobacillus pentosus B96 can be expressed as a function of temperature and $\mathrm{NaCl}$ concentration. Food Microbiol 22: 521-528.
31. Ruiz-Barba JL, Caballero-Guerrero B, Maldonado-Barragan A, JimenezDiaz R (2010) Coculture with specific bacteria enhances survival of Lactobacillus plantarum NC8, an autoinducer-regulated bacteriocin producer, in olive fermentations. Food Microbiol 27: 413- 417.

32. Joshi VK, Sharma S, Rana NS (2006) Production, purification, stability and efficacy of bacteriocin from isolates of natural lactic acid fermentation of vegetables. Food Technol Biotechnol 44: 435-439.

33. Masuda T, Kimura M, Okada S, Yasui H (2010) Pediococcus pentosaceus Sn26 inhibits IgE production and the occurrence ofovalbumin-induced allergic diarrhoea in mice. Biosci Biotechnol Biochem 74: 329-335.

34. Cho YM, Kwak SH, Noh GM, Om AS (2014) Cancer preventative potential of kimchi lactic acid bacteria (Weissella cibaria, Lactobacillus plantarum). J Cancer preven 19: 253-258.

35. Park KY, Jeong JK, Lee YE, Daily JW (2014) Health benefits of kimchi (Korean fermented vegetables) as a probiotic food. J Med Food 17: 6-20.

36. Iyer C, Kosters A, Sethi G, Kunnumakkara AB, Aggarwal BB, et al. (2008) Probiotic Lactobacillus reuteri promotes TNF induced apoptosis in human myeloid leukemiaderived cells by modulation of NF kappa B and MAPK signalling. Cell Microbiol 10: 1442-1452.

37. Kim Y, Oh S, Yun HS, Oh S, Kim SH (2010) Cellbound exopolysaccharide from probiotic bacteria induces autophagic cell death of tumour cells. Lett Appl Microbiol 51: 123-130.

38. Baldwin C, Millette M, Oth D, Ruiz MT, Luquet FM, et al. (2010) Probiotic Lactobacillus acidophilus and L. casei mix sensitize colorectal tumoral cells to 5fluorouracilinduced apoptosis. Nutr Cancer 62: 371-378.

39. Galdeano CM, de Moreno de LeBlanc A, Vinderola G, Bonet ME, Perdigon G (2007) Proposed model: mechanisms of immunomodulation induced by probiotic bacteria. Clin Vaccine Immunol 14: 485-492.

40. Trotta F, Caldini G, Dominici L, Federici E, Tofalo R, et al. (2012) Food borne yeasts as DNA-bioprotective agents against model genotoxins. Inter J Food Microbiol 153: 275-280.

41. Martin MJ, Hulley SB, Browner WS, Kuller LH, Wentworth D (1986) Serum cholesterol, blood pressure and mortality: implications from a cohort of 361, 662 men. Lancet 2: 933-936.

42. Kim HJ, Kwon MJ, Seo JM (2004). The effect of 3 (4' hydroxyl 3'-5'dimethoxyphenyl) propionic acid in Chinese cabbage kimchi on lowering hypercholesterolemia. J Korean Soc Food Sci Nutr 33: 52-58.

43. Miettinen TA, Vanhanen H (1994) Dietary sitosterol related to absorption, synthesis and serum level of cholesterol in different apolipoprotein E phenotypes. Atherosclerosis 105: 217-226.

44. Sheela CG, Augusti KT (1993) Effects of Sallyl cysteine sulfoxide isolated from Allium sativm Linn and gugulipid on some enzymes and fecal excretions of bile and acids and sterols in cholesterol fed rats. Ind J Exp Biol 33: 749-751

45. Kawada T, Hagihara K, Iwai K (1986) Effects of capsaicin on lipid metabolism in rats fed a high fat diet. J Nutr 116: 1272-1278.

46. Taranto MP, Sesma F, Holdago APR, Valdez GF (1997) Bile salts hydrolase plays a key role on cholesterol removal by Lactobacillus casei. Biotechnol Lettr 19: 845-847.

47. Elmadfa I, Klein P, Meyer AL (2010) Immune-stimulating effects of lactic acid bacteria in vivo and in vitro. Proc Nutr Soc 69: 416-420.

48. Chang CK, Wang SC, Chiu CK, Chen SY, Chen ZT, et al. (2015) Effect of lactic acid bacteria isolated from fermented mustard on immune potentiating activity. Asian pacific J Trop Biomed 5: 281-286.

49. Jonganurakkun B, Wang Q, Xu SH, Tada Y, Minamida K, et al. (2008) Pediococcus pentosaceus NB-17 for probiotic use. J Biosci Bioeng 106: 69-73.

50. Won TJ, Kim B, Oh SE, Bang JS, Lee YJ, et al. (2011) Immunomodulatory activity of Lactobacillus strain isolated from fermented vegetables and infant stool. Cand J Physiol Pharm 89: 429-434.

51. Park CW, Youn M, Jung YM, Kim H, Jeong Y, et al. (2008) New functional probiotic Lactobacillus sakei probio 65 alleviates atopic symptoms in the mouse. J Med Food 11: 405-412. 